\title{
Methodological improvement of publications in the JIMD
}

\author{
Better is the enemy of good - Voltaire
}

Peter Burgard

Published online: 2 July 2010

(C) SSIEM and Springer 2010

As an author for the JIMD, I have enjoyed the gradual increase in the impact factor (now 3,598) of the journal. Also as a communicating editor and a reviewer, I feel obliged to look for places for further improvement. As a social scientist and a neuropsychologist, my interests may only partly coincide with those of medical and natural scientists investigating inherited diseases, but all of us share an interest in methodological stringency and clarity.

It cannot be the aim of an editorial to give an introduction to research methodology and statistics in the metabolic sciences (there are excellent publications on this, e.g., Jones and Payne 1997; Holmes 2004), but it can be the aim to make authors and readers more aware of methodological and statistical arguments. The following list of recommendations, although far from complete, describes 10 problems that can often be observed in manuscripts and publications - problems that can be easily solved, thereby improving the quality of the manuscripts submitted and articles published in the JIMD.

1. Hypotheses should always be tested. For each test, formal criteria must be specified and test results should be interpreted and discussed.

2. Most often these tests are based on statistical algorithms. In those cases conventions of statistical reporting should be accepted, including the name of the test, test statistics, $p$-values, and where necessary and possible, basic parameters. For example a comparison of two means by a $t$-test should report

Communicated by: Verena Peters

Competing interest: None declared.

P. Burgard $(\square)$

Department of Pediatric and Adolescent Medicine,

Im Neuenheimer Feld 430,

69120 Heidelberg, Germany

e-mail: Peter.Burgard@med.uni-heidelberg.de the two means, the corresponding standard deviations and samples sizes or degrees of freedom as well as the values for $t$ and $p$.

3. One-sided and two-sided tests should be appropriately chosen. Two-sided tests are made for situations when you think there might be a difference between groups, but you have no idea whether values measured in group A will be lower or higher than those measured in group B. In most studies published in this journal, two-sided tests are inappropriate as, in general, samples from individuals with a (severe, untreated) metabolic disorder are compared with samples from individuals with no (or mild, treated) disorder, and biochemistry and pathophysiology suggest a particular direction for the difference between the two samples.

4. Decide carefully whether sets of measurements are dependent (e.g. coming from the same sample in a repeated measurement design, or from two samples that are comparable for at least some other important aspects) or independent (e.g. for one of the groups you have only the measurement of your key variable and no further information). Statistical tests for dependent measures have a greater power to detect differences and therefore to produce significant results.

5. Avoid what Abelson (1995) calls hocus focus. If your research design has included more than one independent and/or dependent variable, do not only report selected results but choose appropriate multifactorial (more than one independent variable) or multivariate (more than one dependent variable) tests. If you use advanced statistical methods, assist the reader by giving a short explanation of what the method does and a reference.

6. Report at least verbally what you do not report in the statistical analysis, and report nonsignificant results in the same way as you report significant results (see no. 2).

7. In the statistical model mostly used in the JIMD, a significant result means that the probability that the 
result could have appeared by chance is below a predefined threshold (e.g. 0.05, 0.01). There are good reasons not to report only that $p$ is equal to or less than the threshold (significance) or greater than the threshold (nonsignificance). In many cases it would be more appropriate to report the exact probability of a given result or even better the confidence interval (Cohen 1994; Gigerenzer 1993), or as Rosnow and Rosenthal (1989) have written "God loves 0.06 nearly as much as 0.05." However, this must also be accepted by reviewers. Some will criticise statements such as "results approached significance," reminding the author of the threshold rule; others will criticise rejection of results slightly above the threshold as nonsignificant, demanding the precise $p$-value.

8. Retrospective designs or historical data? There is a quite common misconception that going back to historical data (files or databases) is equivalent to a retrospective design. However, in a retrospective design you are looking from the dependent variable (the effect) to the independent variables (the cause). For example, in a study investigating possible causes for mild or severe metabolic phenotypes, one can (retrospectively) compare the genotypes of the severe phenotypes with those of the mild phenotypes. If the question is, for example, about the metabolic phenotypes of different genotypes and existing data are retrieved from patients' files, the analysis is prospective (i.e. looking from the cause to the effect), but the data are historical.

9. Parsimoniousness is a criterion for the design of a study as well as for the study report. The most frequent violation is redundancy in manuscripts where text, figures and tables unnecessarily repeat the same information.

10. Closely related to the last mentioned aspect is coherence of a manuscript. A final check of a manuscript should always be reserved to formal aspects. In the abstract, but not only there, aims and conclusions should refer to one another.

A problem inherent in research on inherited metabolic diseases is the low frequency in cases of orphan diseases resulting in very small sample sizes and low statistical power, and even multicentre studies may have difficulties solving this problem. Therefore, it may be wise to start with problems where fast and easy solutions are available.

\section{References}

Abelson R (1995) Statistics as principled argument. Erlbaum, Hillsdale, $\mathrm{NJ}$

Cohen J (1994) The Earth Is round ( $\mathrm{p}<.05)$. Am Psychol 49:9971003

Gigerenzer G, (1993) The superego, the ego, and the id in statistical reasoning. In: Keren $\mathrm{G}$ and Lewis $\mathrm{C}$ (eds) A handbook for data analysis in the behavioral sciences: methodological issues. Erlbaum, Hillsdale, NJ pp 311-339

Holmes TH (2004) Ten categories of statistical errors: a guide for research in endocrinology and metabolism. Am J Physiol Endocrinol Metab 286:495-501

Jones GJ, Payne RB (1997) Clinical investigations and statistics in laboratory medicine. ACB Venture, London

Rosnow RL, Rosenthal R (1989) Statistical procedures and the justification of knowledge in psychological science. Am Psychol $44: 1276-1284$ 